\section{Patient-Reported Outcomes and Quality of Life Assessment: New Targets for New Targeted Therapy?}

We read with great interest the article by Wood et al, ${ }^{1}$ which demonstrated that patient-reported physical component impairment reported before hematopoietic cell transplantation (HCT) independently predicted poor overall survival after allogeneic HCT. Prompted by their article, we would like to provide some interesting observations.

First, the physical component scale of the Medical Outcomes Study Short Form-36 Health Survey (SF-36) questionnaire enumerates 4 components: physical functioning, role functioning, bodily pain, and general health. The patient-perceived component impairment could be related to coexisting illness (hematological disease burden, therapy-related complications, or comorbid illness), as stated by the authors. It also could be considered as a causal factor for complications, with a subsequent effect on morbidity and overall survival. ${ }^{2}$ In our opinion, among the 4 components, physical functioning retains a major role in the prognosticate patient trajectory during all the phases of disease and treatment, from initial treatment to transplant, and from diagnosis to advanced-phase disease. ${ }^{3,4}$

Second, the article by Wood et $\mathrm{al}^{1}$ strengthens our opinion in favor of both the baseline and prospective use of quality of life assessment with the aim of producing a "dashboard" with indicators and alarms ${ }^{5}$ that may both ameliorate the capacity of prognosticate HCT risk prior to the procedure and help to monitor the development of complications after HCT. However, several points should be discussed concerning this issue, such as which symptoms should be evaluated (predefined symptoms, patienttailored symptoms) and how often (daily, weekly, monthly), but suggestions could be drafted. ${ }^{6}$

Finally, patient-reported outcomes assessment could be interlaced with prearranged patient-reported outcomesdriven procedures of supportive care, such as prehabilitation/rehabilitation or pain control. ${ }^{7}$ It is our firm opinion that those targeted interventions should be initiated

The authors of the original article were invited to respond but declined to do so. promptly to prevent and treat physical component impairment, not only at the time of transplantation but immediately after disease diagnosis and during induction therapy. Effects on both the patients' quality of life and caregivers' workload are strongly expected, whereas a positive impact on morbidity and mortality could only be hypothesized and addressed with dedicated clinical trials.

\section{FUNDING SUPPORT}

No specific funding was disclosed.

\section{CONFLICT OF INTEREST DISCLOSURES}

The authors made no disclosures.

\section{REFERENCES}

1. Wood WA, Le-Rademacher J, Syrjala KL, et al. Patient-reported physical functioning predicts the success of hematopoietic cell transplantation (BMT CTN 0902). Cancer. 2016;122:91-98.

2. Tendas A, Niscola P, Scaramucci L, et al. Motor disability, clinical complications, quality of life, and survival in patients with cancer: a plausible scenario. Support Care Cancer. 2014;22:2603-2604.

3. Efficace F, Cartoni C, Niscola P, et al. Predicting survival in advanced hematologic malignancies: do patient-reported symptoms matter? Eur J Haematol. 2012;89:410-416.

4. Tendas A, Niscola P, Venditti D, et al. Disability and survival of home care onco-hematological patients. Haematologica. 2015;100: 37.

5. Tendas A, Niscola P, Scaramucci L, et al. Making quality of life assessment a dashboard for patient management. Support Care Cancer. 2014;22:2311-2312.

6. Tendas A, Pignatelli AC, Sollazzo F, et al; Rome Transplant Network. Quality-of-life monitoring during hematopoietic stem cell transplantation: observations and a suggestion from the Rome Transplant Network Quality of Life Working Party. J Pain Symptom Manage. 2013; 45:e2-e3.

7. Niscola P, Romani C, Scaramucci L, et al. Pain syndromes in the setting of haematopoietic stem cell transplantation for haematological malignancies. Bone Marrow Transplant. 2008;41:757-764.

Andrea Tendas, MD Department of Hematology S. Eugenio Hospital Rome, Italy

Luca Cupelli, MD Department of Hematology S. Eugenio Hospital Rome, Italy Maria Rita Mauroni, RD Stem Cell Transplant Unit Department of Hematology Tor Vergata University Rome, Italy Fabio Sollazzo, RD Department of Hematology S. Andrea Hospital Rome, Italy

Fabio Di Piazza, STA Stem Cell Transplant Unit Department of Hematology Tor Vergata University Rome, Italy 
Debora Saltarelli, RD

Department of Hematology

S. Giovanni Addolorata Hospital Rome, Italy

Ilaria Carli, RD

Department of Hematology

S. Giovanni Addolorata Hospital Rome, Italy

Anna Chierichini, MD

Department of Hematology

S. Giovanni Addolorata Hospital Rome, Italy

Chiara Melfa, RD

Department of Hematology

University Campus Bio-Medico

Rome, Italy

Maria Antonietta Surano, RD

Department of Hematology

University Campus Bio-Medico

Rome, Italy

Ombretta Annibali, MD

Department of Hematology

University Campus Bio-Medico Rome, Italy

Monica Piedimonte, MD

Department of Hematology

S. Andrea Hospital

Rome, Italy

Esmeralda Conte, MD

Department of Hematology

S. Andrea Hospital

Rome, Italy

Francesco Marchesi, MD

Department of Hematology

Regina Elena National Cancer Institute Rome, Italy

Caterina Viggiani, RD

Department of Hematology

Regina Elena National Cancer Institute Rome, Italy

Adriana Concetta Pignatelli, RD

Department of Hematology

Regina Elena National Cancer Institute

Rome, Italy

Teresa Dentamaro, MD

Department of Hematology

S. Eugenio Hospital

Rome, Italy

Paolo de Fabritiis, PROF

Department of Hematology

S. Eugenio Hospital

Rome, Italy

Alessio Pio Perrotti, MD

Department of Hematology

S. Eugenio Hospital Rome, Italy

William Arcese, PROF

Stem Cell Transplant Unit Department of Hematology

Tor Vergata University Rome, Italy

On behalf of the Quality of Life Working Party of the

Rome Transplant Network

DOI: 10.1002/cncr.29943, Published online March 11, 2016 in Wiley Online Library (wileyonlinelibrary.com)

\section{Percentage of Colorectal Cancer Diagnosed in Adults Aged Younger Than 50 Years}

We read with interest the article by Abdelsattar et al on colorectal cancer (CRC) treatment patterns and outcomes in adults aged $<50$ years. ${ }^{1}$ CRC is a major concern in this age group because incidence rates are increasing, in contrast to rapid declines noted in those aged $\geq 50$ years largely due to the rapid uptake of colonoscopy over the past decade. ${ }^{2}$ In addition, younger patients are more often diagnosed at a late stage of disease due to a lack of timely follow-up of symptoms and because they have not reached the recommended age at which to begin screening. One factor that contributes to the age at which screening should begin is disease age distribution. Abdelsattar et al concluded that $15 \%$ of CRC diagnoses occur in patients aged $<50$ years based on incident cases in Surveillance, Epidemiology, and End Results registries from 1998 through 2011 among individuals aged 20 to 79 years. However, there are 2 biases in their calculation. The most egregious is the unexplained exclusion of patients aged $\geq 80$ years at the time of diagnosis, who represented $24 \%$ of CRC cases in this cohort. ${ }^{3}$ Second, patients that had any previous or subsequent cancer diagnosis, representing approximately $10 \%$ of cases aged 50 to 79 years, also were excluded. ${ }^{4}$ The actual percentage of microscopically confirmed CRC cases diagnosed among individuals aged 20 to 49 years in the 13 Surveillance, Epidemiology, and End Results registries that collected data continuously between 1998 and 2011 was 9.7\% (23,970/247,865). Perhaps more interesting is that this percentage increased over time, from $8.3 \%$ in 1998 to $11.6 \%$ in 2011 , despite the fact that the percentage this age group represented in the adult population decreased from $65.3 \%$ to $57.7 \%$.

The American Cancer Society recommends that CRC screening begin at age 50 years for those at average risk. However, individuals who have a first-degree relative (mother, father, sibling, or child) with a history of CRC or adenomatous polyps should begin screening at age 40 years or 10 years before the youngest case was diagnosed, whichever is sooner. ${ }^{5}$ Patients with chronic inflammatory bowel disease or genetic syndromes that predispose to colorectal cancer, such as Lynch syndrome, also should begin screening before age 50 years. It is incumbent on the public health community to do a better job of informing Americans about when early screening is appropriate. 